www.jmscr.igmpublication.org

Impact Factor (SJIF): 6.379

Index Copernicus Value: 71.58

ISSN (e)-2347-176x ISSN (p) 2455-0450

crossrefDOI: https://dx.doi.org/10.18535/jmscr/v6i6.184

Journal Of Medical Science And Clinical Research

IGM Publication

An Official Publication of IGM Publication

\title{
Comparison between the Effects of Superficial Abdominal and Core Muscle Strengthening on Hemodynamic Parameters in Females with Body Mass Index more than Twenty Five
}

\author{
Authors \\ Dr Shradha Sawant Deshpande ${ }^{1}$, Ms. Niramayee Prabhu ${ }^{2}$ \\ MGM College of Physiotherapy, MGM Hospital, Kamothe Navi Mumbai, India \\ Corresponding Author \\ Dr Shradha Sawant Deshpande \\ Email: shradhasawant18@gmail.com, Contact Info +919987154918
}

\begin{abstract}
It has been proved that obese population shows a reduced strength of the abdominal muscles and core muscles. Studies have also shown that obese population have a reduced abdominal strength and increased of fluctuations in the hemodynamic parameters. There are few studies conducted on the immediate effects of abdominal exercises on hemodynamic parameters in normal young individuals. But, a limited literature was available on the comparison between the core and abdominal exercise as a longterm effect on the immediate changes in these parameters. Hence the purpose of the study was to find the effects of Superficial Abdominal and Core Muscle Strengthening on Hemodynamic Parameters in females with Body Mass Index more than Twenty Five. Nineteen subjects were randomly divided in group A and group B with Body mass Index > 25.Group A underwent superficial muscle strengthening and Group B for core muscle strengthening for 3days/week for 6weeks. Significant improvement was seen in both groups with respect to abdominal strength, all hemodynamic parameters except diastolic Blood pressure (p-value 0.095*) and Waist-Hip Ratio (p-value 0.968*). According to analysis it was concluded that core muscle strengthening is better for stabilizing hemodynamic parameters as compared to superficial abdominal strengthening.

Key words: Overweight, Obese, Core Strengthening, Superficial Abdominals.
\end{abstract}

\section{Introduction}

Obesity is defined as abnormal or excessive accumulation of fat in the body that may impair health. ${ }^{[1]}$ WHO global estimates of 2014 show that $13 \%$ of world population ( $11 \%$ men, $15 \%$ women) who are 18 years $\&$ above are obese ${ }^{[1]}$

It has been found that obese population shows a reduced strength of the abdominal muscles and core muscles. ${ }^{[2]}$ The abdominal muscles from the outside to the inside are external oblique, internal oblique, transversus abdominal and all these muscles merge together at the centre to surround the rectus abdominis. ${ }^{[3]}$

The core includes all the muscles which are present in the area of the belly and the mid and lower back unto the hips including the abdominal muscles. They are divided into local muscles and global muscles depending upon their location 
${ }^{[4]}$ Abdominal muscles are special as they provide postural support. The core muscles on the other hand are mostly acting as stabilizers and force transfer centres. Core training focuses on maintaining even pelvic/spinal posture during exercises. ${ }^{[5]}$ It has been proved in a study by Nelson Nardo that obese population have a reduced abdominal strength and a high risk of cardiovascular issues. Also this study proved that this population shows a lot of fluctuations in the hemodynamic parameters. ${ }^{[6]}$

There is a close link seen between the abdominal strength and the hemodynamic parameters (blood pressure, heart rate, $\mathrm{SpO} 2$ ) which shows an indirect relationship. ${ }^{[7]}$ It is likely that heart rate and blood pressure change during abdominal exercise because large muscle co-contractions are involved, that are expected to increase intraabdominal pressure (IAP) ${ }^{[7]}$. Those who regularly engage in resistance training show less dramatic blood pressure increases than untrained counterpart. ${ }^{[8]}$

Obesity also affects the respiratory system. ${ }^{[9]} \mathrm{A}$ study conducted by $\mathrm{Li} \mathrm{J}$ has shown that there is an increased resting respiratory rate in individuals with increased hip-waist ratio and BMI compared to the ones in a normal category. ${ }^{[10]}$

Although waist circumference and BMI are interrelated, waist circumference provides an independent prediction of risk over and above that of BMI. Waist circumference measurement is useful in patients who are categorized as normal or overweight on the BMI scale. In females, a waist circumference of above $88 \mathrm{~cm}$ is considered a high risk for cardiovascular diseases. ${ }^{[9]}$ There were few studies conducted on the immediate effects of abdominal exercises on hemodynamic parameters in normal young individuals. ${ }^{[7]}$ But, a limited literature is available on the comparison between the core and abdominal exercise as a long-term effect on the immediate changes in these parameters.

So this study was done to find which protocol would be more beneficial in stabilizing the parameters.
Materials and Methodology

Participants: Nineteen subjects were part of the study according to the inclusion and exclusion criteria which consisted of Overweight \& Obese young females (BMI >25), Age: 18 to 25 years and those not under any physical training program for at least 6 months. All the procedures were explained to the subjects and their information was kept confidential.

\section{Materials Used: Sphygmomanometer,} Stethoscope, Pulse Oximeter, Yoga Mat, Measuring tape, Weighing machine, Consent form, Pen, Paper.

\section{Method}

The evaluation of the subjects height, weight, BMI, abdominal muscle strength (using Isometric abdominal strength test $)^{[11]}$ and waist circumference was done. The immediate changes in the hemodynamic parameters (SBP, DBP, HR, and $\mathrm{SpO} 2$ ) and $\mathrm{RR}$ were assessed before and after 3 abdominal exercises (Partial curl-ups, Partial Oblique curl-ups, unilateral leg raises). The subjects were divided into two groups (Group A and Group B). Group A performing superficial abdominal exercises and Group B performing core exercises 3 times a week on alternate days under the supervision of the researcher. After completion of 6-weeksexercise protocol, reevaluation of abdominal muscle strength, waist circumference, and immediate changes in hemodynamic changes were measured. Pre \& Post Exercise Regimen values were compared for both groups by Wilcoxon Signed Ranks Test. The data between the groups was then compared by Mann Whitney's U test. 


\section{Results}

Table 1: Demographic Data

\begin{tabular}{|c|c|c|}
\hline MEAN (SD) & GROUP A & GROUP B \\
\hline AGE (years) & $22 \pm 1.22$ & $22.3 \pm 0.483$ \\
\hline WEIGHT (kg) & $71.3 \pm 8.72$ & $71.6 \pm 6.16$ \\
\hline HEIGHT (m) & $1.57 \pm 0.061$ & $1.6 \pm 0.06$ \\
\hline BMI (kg/m $\left.\mathbf{m}^{\mathbf{2}}\right)$ & $28.9 \pm 3.08$ & $27.9 \pm 2.8$ \\
\hline
\end{tabular}

Table 2: Effect of exercises on Abdominal Muscle Strength

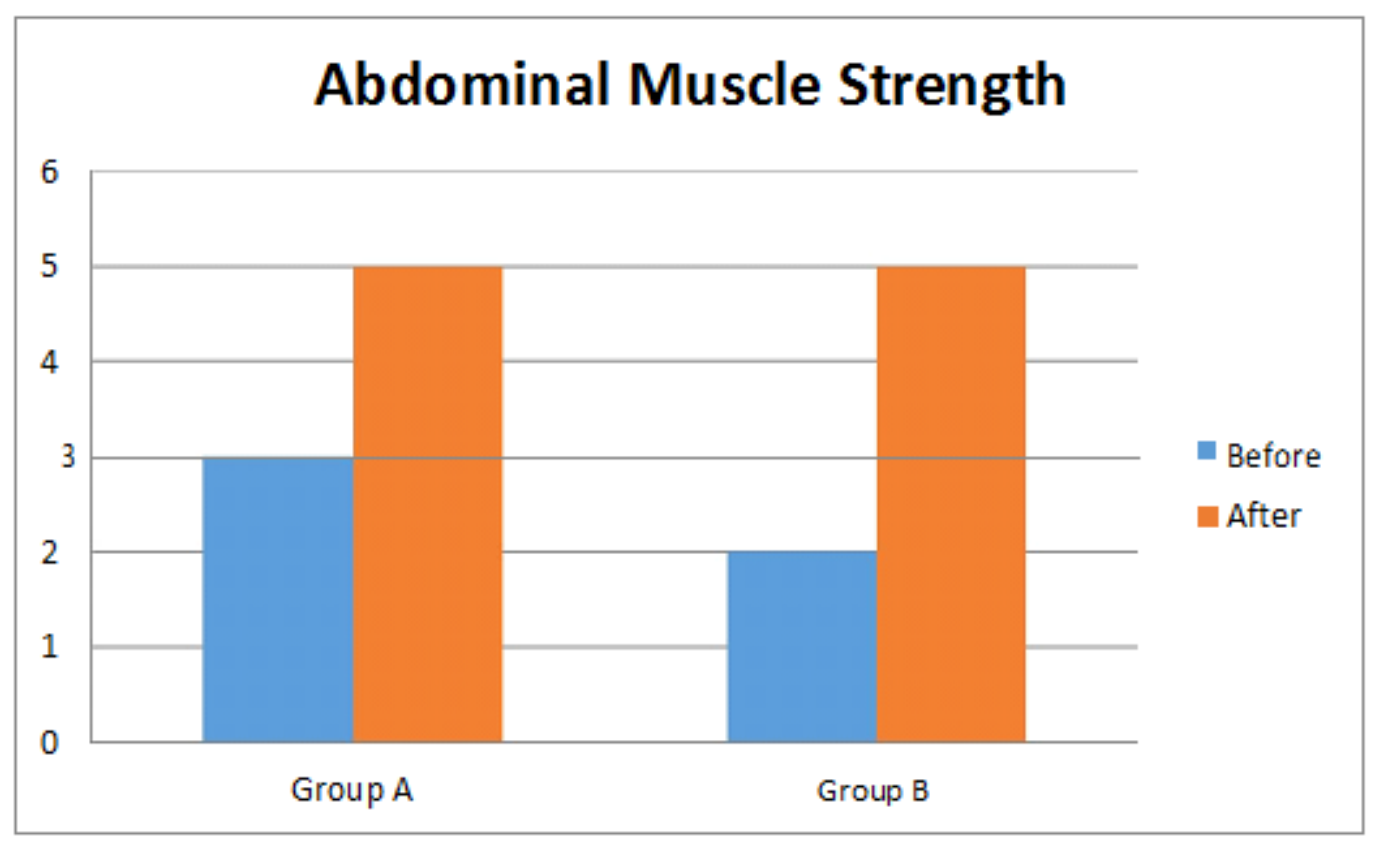

In superficial abdominal strengthening group the In core exercise group the mode of power before mode of power before the exercise was 3 (Fair) the exercise was 2 (Poor) while after the protocol while after the protocol the power was 5 was 5 (Normal)

(Normal).

Table 3: Effect of Exercises on change in Systolic Blood Pressure

\begin{tabular}{|c|c|c|c|}
\hline Variables & Mean \pm SD & Mean \pm SD & \multirow{2}{*}{ P- value } \\
\cline { 2 - 3 } & Pretest & Post test & \multirow{2}{*}{} \\
\hline Group A & $3.33 \pm 2.54$ & $2 \pm 2.6$ & $0.048^{*}$ \\
\hline Group B & $3.60 \pm 2.63$ & $0.60 \pm 2.12$ & $0.031^{*}$ \\
\hline
\end{tabular}

There is a significant difference seen between the post values of Group A and B: p value 0.028* 
Table 4: Effect of Exercises on change in Diastolic Blood Pressure

\begin{tabular}{|l|cc|c|c|}
\hline Variables & \multicolumn{2}{|c|}{ Mean \pm SD } & Mean \pm SD & \multirow{2}{*}{ P - value } \\
\cline { 2 - 4 } & \multicolumn{2}{|c|}{ Pretest } & Post test & \\
\hline Group A & 0.89 & \pm 1 & $0.89 \pm 1.45$ & $1.000^{*}$ \\
\hline Group B & 1.20 & \pm 0.94 & $1 \pm 0.84$ & $0.792^{*}$ \\
\hline
\end{tabular}

There is no significant difference seen between the post values of Group A and B: $p$ value $0.095^{*}$

Table 5: Effect of Exercises on change in Heart Rate

\begin{tabular}{|l|c|c|c|}
\hline Variables & Mean \pm SD & Mean \pm SD & P value \\
\cline { 2 - 3 } & Pretest & Post test & \\
\hline Group A & $9.89 \pm 5.82$ & $6.78 \pm 1.79$ & $0.021^{*}$ \\
\hline Group B & $9.10 \pm 3.54$ & $4.90 \pm 1.73$ & $0.012^{*}$ \\
\hline
\end{tabular}

There is a significant difference seen between the post values of Group A and B: p value $0.043^{*}$

Table 6: Effect of Exercises on change in Respiratory Rate

\begin{tabular}{|l|rr|c|c|}
\hline \multirow{2}{*}{} & \multicolumn{2}{|c|}{ Mean \pm SD } & Mean \pm SD & \multirow{2}{*}{ P value } \\
\cline { 2 - 4 } & \multicolumn{2}{|c|}{ Pretest } & Post test & \\
\hline Group A & 6.33 & \pm 1.94 & $4.22 \pm 1.09$ & $0.007 *$ \\
\hline Group B & $5.70 \quad \pm 2$ & $2.90 \pm 2.18$ & $0.012^{*}$ \\
\hline
\end{tabular}

There is a significant difference seen between the post values of Group A and Group B: p value 0.043*

Table 7: Effect of Exercises on change in SPO2

\begin{tabular}{|l|c|c|c|}
\hline Variables & Mean \pm SD & Mean \pm SD & \multirow{2}{*}{ P value } \\
\cline { 2 - 3 } & Pretest & Post test & \multirow{2}{*}{} \\
\hline Group A & $1.67 \pm 1.27$ & $0.67 \pm 0.87$ & $0.171^{*}$ \\
\hline Group B & $1.50 \pm 0.71$ & $0.90 \pm 1.10$ & $0.141^{*}$ \\
\hline
\end{tabular}

There is no significant difference seen between the post values of Group A and Group B: p value 0.780*

Table 8: Effect of Exercises on Waist Circumference

\begin{tabular}{|l|c|c|c|}
\hline Variables & Mean \pm SD & Mean \pm SD & \multirow{2}{*}{ P value } \\
\cline { 2 - 3 } & Pretest & Post test & \multirow{2}{*}{} \\
\hline Group A & $90.40 \pm 7$ & $89.10 \pm 7.05$ & $0.008^{*}$ \\
\hline Group B & $86.20 \pm 5.79$ & $84.89 \pm 6.12$ & $0.005^{*}$ \\
\hline
\end{tabular}

There is no significant difference seen between the post values of Group A and Group B: p value 0.968* 


\section{Discussion}

The study has shown that a 6 week long core exercises and superficial abdominal exercises protocol both has an effect on the immediate changes in hemodynamic parameters, respiratory rate and waist circumference. There was no significant difference seen in the diastolic blood pressure in either of the groups.

Among both the protocols, core exercises have proved to be better in reducing the systolic blood pressure, heart rate and respiratory rate when compared with the superficial strengthening group. But, there was no significant difference observed in the post values of diastolic blood pressure, waist circumference, $\mathrm{SpO} 2$ between the 2 groups.

Increase in muscle strength provide reduction in muscular activation and metabolic demand for the same absolute effort. These adaptations positively reflected on the hemodynamic responses, leading to lower cardiovascular stress during a sub maximal effort. ${ }^{[12]}$

There has been an increase in the systolic blood pressure seen immediately after the exercises done before the 6 week exercise protocol in both groups. This difference reduced after the exercise protocols were given to these individuals. This immediate increase could be due to the fact that straining type exercises increases blood pressure dramatically because sustained muscular force compresses peripheral arterioles, considerably increasing the resistance to blood flow. Those who regularly engage in resistance training show less dramatic blood pressure increases than untrained counterpart. ${ }^{[8]}$ The exercises reduce the muscular compressive forces on the arteriole. Also, the exercise reduce the sympathetic activity and reduce the vascular resistance. This would be the reason that after the exercise protocol there was a decrease in the changes in systolic blood pressure. ${ }^{[8]}$

There was no significant increase in diastolic blood pressure observed immediately and before and after the exercise protocol. This could be due to the isometric component, which may have been insufficient to promote a significant increase in diastolic blood pressure. The second and more likely mechanism responsible for the DBP response is the fact that the measurements were performed immediately after each abdominal exercise. A rebound vasodilatation after each exercise cold also have been the reason for no increase in diastolic blood pressure. ${ }^{[7]}$

There was an increase in the heart rate immediately so as to meet the demands of the muscles (nutrients, oxygen). After exercise the change in heart rate reduced in these subjects as the volume of blood pumped with each beat must have increased with the exercise protocol. Thus, the heart rate reduced as the demand was met in a limited heart rate. As in core strengthening program the muscles required more oxygen supply, this group has shown a better effect in stabilizing the heart rate.

The respiratory rate stabilized after exercises as the need for oxygen was met by the limited respiratory rates. Also the blood supply to the alveoli increased with exercises. Thus, there was an increase in the gas-exchange, thus reducing the respiratory rate. Also with the core strengthening protocol, we also work on strengthening the respiratory muscles, thus improving the volume and capacities of lung. All these help in improving the lung function, and therefore, reduce the respiratory rate.

The saturation of oxygen did not alter as the oxygen consumption and availability may have been balanced well during and after the exercise.

\section{Conclusion}

Through this research we can conclude that the both exercise protocols, i.e. Abdominal and core strengthening exercises help in stabilizing the hemodynamic parameters. But, among the two, core exercise protocol is better at stabilizing these parameters when compared with the superficial abdominal exercise. So the core exercise protocol is better at stabilizing the parameters. 


\section{Acknowledgment}

We would like to extend our sincere gratitude to all our participants who were relentless and very cooperative throughout the study.

\section{References}

1. Obesity and Overweight Fact sheet $\mathrm{N}^{0} 311$. WHO. January 2015. Retrieved 2 February 2016.

2. Abdominal cavity. Encyclopaedia Britannica. I: A-Ak - Bayes (15th ed.). Chicago, IL: Encyclopaedia Britannica, Inc. 2010. pp. 19-20.

3. Carey D.G.P. (1998). Abdominal Obesity. Current Opinion in Lipidology. (pp. 3540). Vol. 9, No Retrieved on April 9, 2012.

4. Karageanes, Steven J. Principles of Manual sports medicine. Lippincott Williams \& Wilkins. 2014 pp. 510-511

5. Shirley Sahrman. Diagnosis and Treatment of Movement Impairment Syndromes. Mosby; 2002.

6. Nelson Nardo Junior. Impact of abdominal obesity on anthropometric, physical and hemodynamic parameters according to sex in adolescent beginning a multidisciplinary intervention program. CMR Journal. 2015; 15-16.

7. Mujumdar S, Ganesh B R. Immediate effect of abdominal exercises on hemodynamic responses in healthy young adults- an experimental study. Indian journal of Physiotherapy and Occupational Therapy.

8. Victor L. Katch, William McArdle, Frank I. Katch. Essentials of Exercise Physiology. $4^{\text {th }}$ edition. Lippinocott Williams \& Wilkins. Pg 307

9. Koenig SM. Pulmonary Complications of obesity. Am J Med Sci. 2001; 321:249279.

10. Burki NK, Baker RW. Ventilatory regulation in eucapnic morbid obesity. Am. Rev. Respir. Dis. 1984; 129: 538-43.

11. Kendall F: Muscles, testing and function, ed 3, Baltimore, 1983, Williams \& Wilkins.

12. Andre Luiz DemantovaGurjao, Raquel Goncalves, et al. Effect of resistance training in blood pressure at rest in normotensive elderly. Rev Bras Med Esporte. 2013, 19(3). 